\title{
Morphometric Relations of Fractal-Skeletal Based Channel Network Model
}

\author{
B.S. DAYA SAGAR *, CHARLES OMOREGIE and B.S. PRAKASA RAO \\ Centre for Remote Sensing \& Information Systems, Department of Geo Engineering, Andhra University, \\ Visakhapatnam 530 003, India
}

(Received 20 February 1997; Revised 29 August 1997)

\begin{abstract}
A fractal-skeletal based channel network (F-SCN) model is proposed. Four regular sided initiator-basins are transformed as second order fractal basins by following a specific generating mechanism with non-random rule. The morphological skeletons, hereafter referred to as channel networks, are extracted from these fractal basins. The morphometric and fractal relationships of these F-SCNs are shown. The fractal dimensions of these fractal basins, channel networks, and main channel lengths (computed through box counting method) are compared with those of estimated length-area measures. Certain morphometric order ratios to show fractal relations are also highlighted.
\end{abstract}

Keywords: Fractal, Morphological skeleton, F-SCNs, Morphometry, River networks

\section{INTRODUCTION}

The stream network is an example of a structure having multiple complexity. Accurate morphological description of such a complex structure using mathematical models is difficult. Mandelbrot (1982) was the first to present stream network, which possesses statistical self-similarity, as a fractal. In the stream network, the high degree of geometrical organization conveys several orders of magnitude in size from the trunk stream to minor twigs. The number of branching orders varies depending on the physiographic set-up of the stream basin. Within the basins, the pattern of stream network is geologically determined which reveals the potential applications of fractals to develop algorithms for modelling drainage morphogenesis.

The aim of this study is twofold.

- To establish that the generated fractal-skeletal based channel networks (F-SCNs) with a center as an outlet (glory hole), follow morphometric and fractal relations that are established/proposed in the context of river networks, and to show that F-SCNs follow Horton's laws of river networks.

* Corresponding author. Fax: 091-0891-555547. 
- To find out the converging points of fractal dimensions of the fractal basins and their corresponding channel networks.

The scheme of this paper is to first present the Horton's laws, and various fractal relations proposed with various river network models. The second section discusses the F-SCN model. Certain mathematical morphological transformations that are needed to implement the skeletonization concept to extract channel network from the generated fractal basin is presented. In the third section, fractal relations of F-SCNs thus generated were verified to test the plausibility of the model to ensure that the model produced a network with properties that are of Hortonian system.

\section{Structure of the Network Model}

To characterise the channel network, the morphometric procedures proposed by Horton (1945) and Strahler (1957) are employed. The Strahler's ordering technique was adopted to designate the orders of channel network to compute certain important dimensionless ratios like bifurcation ratio $\left(R_{\mathrm{B}}\right)$, channel length ratio $\left(R_{\mathrm{L}}\right)$, and area ratio $\left(R_{\mathrm{A}}\right)$.

\section{Strahler's Stream Ordering}

The order of the stream ranges from 1 to $n$. All finger tips are designated as first order streams. Two first order segments unite to form a second order branch. A third order is formed by joining two second order segments, but may be joined by additional first or second order segments. Two third order segments join to form a fourth order segment, and so on. The source streams of order 1 and when two streams of order $i$ and $j$, respectively merge, a stream of order $\omega$ is formed, with

$$
\omega=\max \{i, j, \operatorname{Int}[1+(1 / 2)(i+j)]\},
$$

where function Int[] denotes the integer part of the argument.

\section{List of Symbols, Nomenclature and Morphometric Parameters}

Order of the channel network $\Omega$ of the basin

Number of channel segments $\quad N(\omega)$

of order $\omega$

Channel length of order $\omega \quad L(\omega)$

Area of channel segments of $A(\omega)$

order $\omega$

Total number of channels in $\quad N(\Omega)$

the basin of order $\Omega$

Area of the basin of order $\Omega \quad A(\Omega)$

Mean length of channel $\quad L(\omega, \Omega) / N(\omega, \Omega)$

segments of order $\omega$

Mean area of channel

segments of order $\omega$

Main channel length of

$A(\omega, \Omega) / N(\omega, \Omega)$

$l(\Omega)$

the basin of order $\Omega$

Total length of the channel $L(\Omega)$

network in the basin of

order $(\Omega)$

The fitted exponent between $\quad \beta$

area and total length of

channel network

The fitted exponent between $\quad \alpha$

area and main channel length

Perimeter of the basin

$P$

Circularity ratio $\left(R_{\mathrm{c}}\right)$

$A(\omega, \Omega) / \pi(P / 6.3)^{2}$.

\section{Hortonian River Networks}

In the geomorphological analysis of river networks, scaling properties were defined by Horton's laws (Smart, 1972), Strahler's ordering (e.g. Smart, 1972; Shreve, 1967) yield the bifurcation stream number law.

Bifurcation ratio $\left(R_{\mathrm{B}}\right)$ is the ratio of the number of channel segments of a given order $N(\omega, \Omega)$ to the number of streams to the next highest order, $N(\omega-1, \Omega)(2)$

$$
\frac{N(\omega-1, \Omega)}{N(\omega, \Omega)}=R_{\mathrm{B}}, \quad \omega=2, \ldots, \Omega
$$


Stream length ratio $\left(R_{\mathrm{L}}\right)$ is the ratio of mean length of segments of order $\omega, \bar{L}(\omega, \Omega)$ and mean length of segments of the next lower order, $\bar{L}(\omega-1, \Omega)(3)$ and the stream length law

$$
\frac{\bar{L}(\omega, \Omega)}{\bar{L}(\omega-1, \Omega)}=R_{\mathrm{L}}, \quad \omega=2, \ldots, \Omega,
$$

where $N(\omega, \Omega)$ is the number of streams of order $\omega$ in a basin of order $\Omega(N(\Omega, \Omega=1) ; \bar{L}(\omega, \Omega)$ is the average length of streams of order $\omega$; and $R_{\mathrm{B}}, R_{\mathrm{L}}$ are Horton's numbers of bifurcation and length respectively.

Stream area ratio $\left(R_{\mathrm{A}}\right)$ is the ratio of mean area of segments of order $\omega, \bar{A}(\omega, \Omega)$ and mean area of segments of the next lower order, $\bar{A}(\omega-1, \Omega)(4)$.

The law of stream areas was proposed by Schumm (1956)

$$
\frac{\bar{A}(\omega, \Omega)}{\bar{A}(\omega-1, \Omega)}=R_{\mathrm{A}}, \quad \omega=2, \ldots, \Omega,
$$

where $\bar{A}(\omega)$ is the average area drained by each $\omega$ order stream and $R_{\mathrm{A}}$ is the stream area ratio. The area $A(\omega, \Omega)$ is normally taken to include not only the area drained directly by the particular stream of order $\omega$ but also that drained by all of its tributaries of lower order. The law of stream areas suggests that $R_{\mathrm{A}}>R_{\mathrm{B}}$. This follows from the fact that $A(\omega)>R_{\mathrm{B}} A(\omega-1)$, so that $A(\omega)>\left(R_{\mathrm{B}} / R_{\mathrm{A}}\right) A(\omega)$.

The empirical laws of stream numbers, stream length (Horton, 1945), and stream areas (Schumm, 1956) which enunciate the bifurcation ratio $R_{\mathrm{B}}$, the stream length ratio $R_{\mathrm{L}}$, and the stream area ratio $R_{\mathrm{A}}$ are constant for the homogeneous river basins. Estimates of these ratios can be obtained from the slopes of the straight lines resulting from plots of logarithmic transformed values of $N(\omega, \Omega), L(\omega, \Omega)$, and $A(\omega, \Omega)$ versus of order $\omega$, for $\omega$ ranging from 1 to $\Omega$. Channel network density: The channel density $\rho$ was calculated by following expressions (5)

$$
\rho=L(\Omega) / A(\Omega),
$$

where $A(\Omega)$ and $L(\Omega)$ be the finite measures of the basin area and are of total channel network (i.e., scale dependent).
Channel frequency: Channel frequency, $F$, was calculated by Eq. (6) proposed by Strahler (1957):

$$
F=\sum_{\omega=1}^{\Omega} N(\omega, \Omega) / A(\Omega) .
$$

Melton's law: The Melton's law (Smart, 1972) (7) relates the channel frequency (6) to the square of the channel density (5) with a value of about 0.69 . This law is of interest to test how closely a Horton system obeys Melton's law:

$$
\frac{\sum_{\omega=1}^{\Omega} N(\omega, \Omega) / A(\Omega)}{(L(\Omega) / A(\omega, \Omega))^{2}}=\frac{F}{\rho^{2}} \cong 0.69 .
$$

This law applies for basins of higher order if $R_{\mathrm{B}} \gg R_{\mathrm{L}}$ and $R_{\mathrm{A}} / R_{\mathrm{B}}=1$, or $R_{\mathrm{B}} \ll R_{\mathrm{L}}$ and $\left(R_{\mathrm{B}} R_{\mathrm{A}} /\right.$ $\left.R_{\mathrm{L}}\right)=1$.

There are two proposed methods to test if a model is Hortonian or non-Hortonian. The former is based on Melton's law. Marani et al. (1991), postulate that this law is asymptotically true within a Peono system, which is a non-Hortonian system (Beer and Borgas, 1993). The later method is to examine the channel density. In a non-Hortonian system like the Peono system the channel density does not vary with scale (Marani et al., 1991), whereas in a Horton system it does.

\section{Fractal Description of River Networks}

Several papers have been presented of late which address the fractal description of stream network (La Barbera and Rosso, 1987; Tarbotan et al., 1990; Rosso et al., 1991; Marani et al., 1991; Masek and Turcotte, 1993). These researchers proposed various derivations, based on heuristic arguments to compute fractal dimensions by considering bifurcation and length ratios and certain lengtharea measures. However, the river network models developed by various researchers some relationships have been constituted by means of showing certain similarities based on morphometric parameters as well as fractal and multi-fractal measures. 
The fractal-skeletal based channel network (F-SCN) model is an extension of the previous work (Sagar, 1996) in which preliminary study has been carried out to show certain fractal relationships.

Length-area measures: The dependence of the river areas $(A)$, river lengths $(L)$, and basin perimeter $(P)$, on the Strahler (1957) order can be measured for the river network models. A large number of power law relationships between the characteristics of river networks have been proposed. The exponents of $\alpha, \beta$ may be estimated through the length-area relations (8)-(10). The fractal dimension of the main channel length was computed using length-area measures. The main channel length, $l$ and area, $A$, are related (8) as follows:

$$
l \sim A^{\alpha} .
$$

Hack (1957) found that the above equation described quite well the relationship between the area and length of a river in Virginia and Maryland and that the exponent $\alpha$ had a value of about 0.6. Since river basins have a compact geometry the above equation (8) suggests that the main steam length of a river can be described as a fractal with a dimensionality, $d$ (9) given by Mandelbrot (1982):

$$
d=2 \alpha .
$$

The area and total stream length appear to be related by (10),

$$
L \sim A^{\beta},
$$

where $L$ denotes the total length of streams in the basin of area $A$. $\beta$ is a fitted exponent.

\section{Fractal Measures based on Morphometric Order Ratios}

La Barbera and Rosso $(1987,1989)$ based on intuitive arguments showed the fractal dimension $\left(D_{1}\right)$ of stream networks to be as proposed in
Eq. (11)

$$
\begin{aligned}
& D_{1}=\log R_{\mathrm{B}} / \log R_{\mathrm{L}}, \quad R_{\mathrm{B}} \geq R_{\mathrm{L}}, \\
& D_{1}=1, \quad R_{\mathrm{B}}<R_{\mathrm{L}} .
\end{aligned}
$$

Commenting on the work of La Barbera and Rosso (1987), Tarbotan et al. (1990) proposed a new derivation (12) as follows:

$$
D_{2}=d\left(\log R_{\mathrm{B}} / \log R_{\mathrm{L}}\right),
$$

where $d=$ fractal dimension of the main stream length. Feder (1988) also indicated that the fractal dimension and main river length should be related to the bifurcation ratio $\left(R_{\mathrm{B}}\right)$, length ratio $\left(R_{\mathrm{L}}\right)$ and stream area ratio $\left(R_{\mathrm{A}}\right)$ of the river networks respectively. This is expressed as

$$
\begin{array}{ll}
D_{3}=2\left(\log R_{\mathrm{L}} / \log R_{\mathrm{B}}\right), & R_{\mathrm{B}} \geq R_{\mathrm{A}}, \\
D_{3}=2\left(\log R_{\mathrm{L}} / \log R_{\mathrm{A}}\right), & R_{\mathrm{B}}<R_{\mathrm{A}} .
\end{array}
$$

Rosso et al. (1991) introduced the definition of $D_{4}$ (14) as follows:

$$
\begin{aligned}
& D_{4}=2 \frac{\log R_{\mathrm{B}}}{\log R_{\mathrm{A}}}, \quad R_{\mathrm{B}} \geq R_{\mathrm{A}}, \\
& D_{4}=2, \quad R_{\mathrm{B}}<R_{\mathrm{A}} .
\end{aligned}
$$

Stark (1991) heuristically argued that $D_{4}$ can have no hydrological relevance to a Horton system because it implies an area that tends to infinity as the order tends to infinity. However, it is true that any river basin with finite area as the resolution is refined, though the total length may diverge.

\section{FRACTAL-SKELETAL BASED CHANNEL NETWORK (F-SCN) MODEL}

The stream network is the pattern of stream branches that is determined by inequalities of outline of the basin. The wrinkles in the basin outline may be visualised as topographic undulations. These undulations are treated as the crenulations in the elevation contours which are the flow 
paths of streams. It implies that the number of crenulations on the top most contour in the catchment is equal to number of first orders. After flowing to a certain distance, the two first orders join together to form second order of which the flow path is another crenulation, larger than that of the previous order crenulation of the contour. The stream network, between trunk stream and twigs, exhibits self-similarity and asymmetry. The two tributary branches arising at a stream segment bifurcation often differs in width, length and angle, leading to a highly heterogeneous structure. The tools involved to generate F-SCNs are generating mechanism, which includes the deterministic quantities, like bifurcation ratio, stream length ratio, angle of divergence, the concept of skeletonization, and the rule (random or non-random). This model includes the following two sequential steps:

Step 1. Fractal basin generation;

Step 2. Channel network extraction from the fractal basin.

\section{Fractal Basin Generation}

To generate fractal basins with a fractal dimensions range from 1 to 2 in 2-dimensional space, one begins with two shapes, (1) broad outline of the basin as polygon, an initiator-basin, and (2) a generator. The latter is an oriented broken line made up of $N$ equal sides of length $r$ which can be designed at will (Mandelbrot, 1982). Each stage of the construction begins with a broken line and consists in replacing each straight interval with a copy of the generator, reduced and displaced to have the same end points as those of the interval being replaced. In all cases, $D=\log N / \log 1 / r$. Step 0 is to draw the segment of length $(0,1)$, which is one side length in the initiator-basin. Step 1 is to draw either of the kinked curves (Fig. 1), each made up of $N$ intervals superposable upon the segment $(1 / 4,1 / 3,1 / 3$ for the generator shown in Fig. 1). Step 2 is to replace each of the $N$ segments used in step 1 by a kinked curve obtained by reducing the curve of step 1 in the ratio $r(N)=1 / r$. One obtains altogether $N^{2}$ segments of length $1 /(r)^{2}$. Iterating this process adds further detail. Homogeneous and heterogeneous channel network patterns will be determined respectively by symmetric generator with non-random, and asymmetric generator with random rules. Asymmetric fractal basins arise due to asymmetric outline of the initiator and also due to generating mechanism and the rule applied to transform the initiator as fractal basin. The boundary of the fractal basin possesses many crenulations that have been taken as the basis to find out the flow paths of the stream network. These undulations in the outline of the fractal basin and in the successive bifurcation generation levels determine the whole stream network pattern. The fractal basin can be transformed as channel network, a line thinned caricature to summarise the shape, size, orientation and connectivity of the basin.

To implement the second step, certain mathematical morphological transformations (Serra, 1982) were employed. These transformations were used to implement the concept of skeletonization proposed
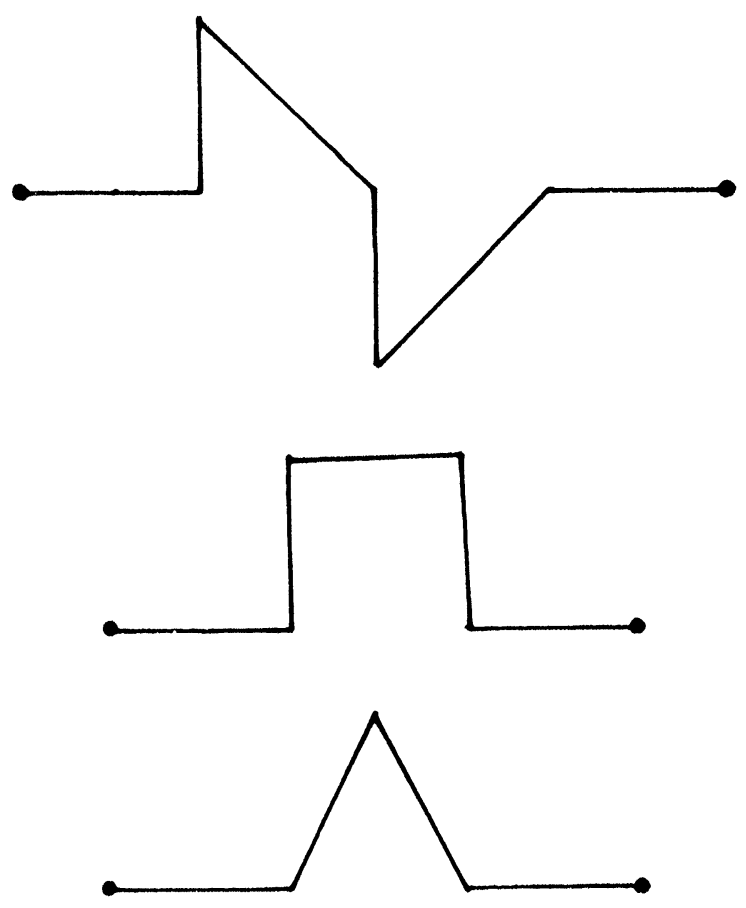

FIGURE 1 Different generators. 
by Lantuejoul (1980). The concept of skeletonization which is considered as the basis to extract the channel network in this present investigation was proposed by mathematical morphologists (Serra, 1982; Lantuejoul, 1978; 1980; Maragos and Schafer, 1986). This concept is being extensively applied in several fields such as biological shape description (Blum, 1973), pattern recognition (Maragos and Schafer, 1986), metallography (Lantuejoul, 1978; 1980) with highly promising results. Certain important morphological transformations that are essential to implement skeletonisation process are briefly described as follows:

\section{Mathematical Morphological Transformations}

Mathematical morphology based on set theoretic concepts is one the approaches to the analysis of geometric properties of different structures. The fractal basin, $M$, a discrete binary image is defined as a finite subset of Euclidean two-dimensional space, $Z^{2}$. The geometrical properties of a fractal basin as a set $(M)$ and set complement $\left(M^{\mathrm{c}}\right)$ are subjected to the morphological functions. The morphological operators is visualised as working with two images. The image being processed is referred to as the fractal basin that needs to be decomposed into channel subsets, and the other image being a structuring template $(S)$. Each structuring template possesses a designed shape that is thought of as a probe of fractal basin. In addition, many structuring templates were represented by a compact subset of Euclidean space, so that constraints which correspond to the four principles of the theory of mathematical morphology such as invariance under translation, compatibility with change of scale, local knowledge and uppersemicontinuty will be imposed on morphological set transformations (erosion, dilation, opening and closing), for precise extraction of topological information from the drainage network extraction point of view. The three morphological transformations involved in drainage network extraction are dilation to expand, erosion to shrink, and cascade of erosion-dilation to smoothen the set. These transformations are based on Minkowski set addition and subtraction (Hadwiger, 1957).

Dilation: Dilation combines two sets using vector addition of set elements. If $M$ and $S$ are sets in Euclidean space with elements $m$ and $s$, respectively, $m=\left(m_{1}, \ldots, m_{N}\right)$ and $s=\left(s_{1}, \ldots, s_{N}\right)$ being $N$-tuples of element co-ordinates, then the dilation of $M$ by $S$ (structuring template) is the set of all possible vector sums of pairs of elements, one coming from $M$ and the other from $S$. The dilation of a fractal basin, $M$, with structuring template, $S$, is defined as the set of all points ' $m$ ' such that $S_{m}$ intersects $M$. It is expressed as

$$
M \oplus S=\left\{m: S_{m} \cap M \neq \emptyset\right\}=\bigcup_{s \in S} M_{s} .
$$

Erosion: The erosion of a fractal basin, $M$, with structuring template, $S$, is defined as the set of points ' $m$ ' such that the translated $S_{m}$ is contained in $M$. It is expressed as

$$
M \ominus S=\left\{m: S_{m} \subseteq M\right\}=\bigcap_{s \in S} M_{s},
$$

where $S=\{s: s \in S\}$, i.e. $S$ rotated $180^{\circ}$ around the origin. For more information on mathematical morphological transformations, reader may refer to Serra (1982).

\section{Channel Network Extraction}

Channel network, a line thinned caricature to summarise the shape, size, orientation and connectivity of the basin is extracted from fractal basin. Components of such networks include traditional characteristics of shape in two-dimensional space. The channel network of a basin (Fig. 2(a)) viewed as a subset of $R^{2}$ (Euclidean space), is defined as the set of the centers of the maximal disks inscribable inside the basin (Fig. 2(b)). Figure 2(c) is the extracted channel network that can be arrived at through the procedure described in Eqs. (17) and (18). The channel network, $\mathrm{CH}(M)$ of a fractal basin $(M)$ viewed as a subset of $R^{2}$ 

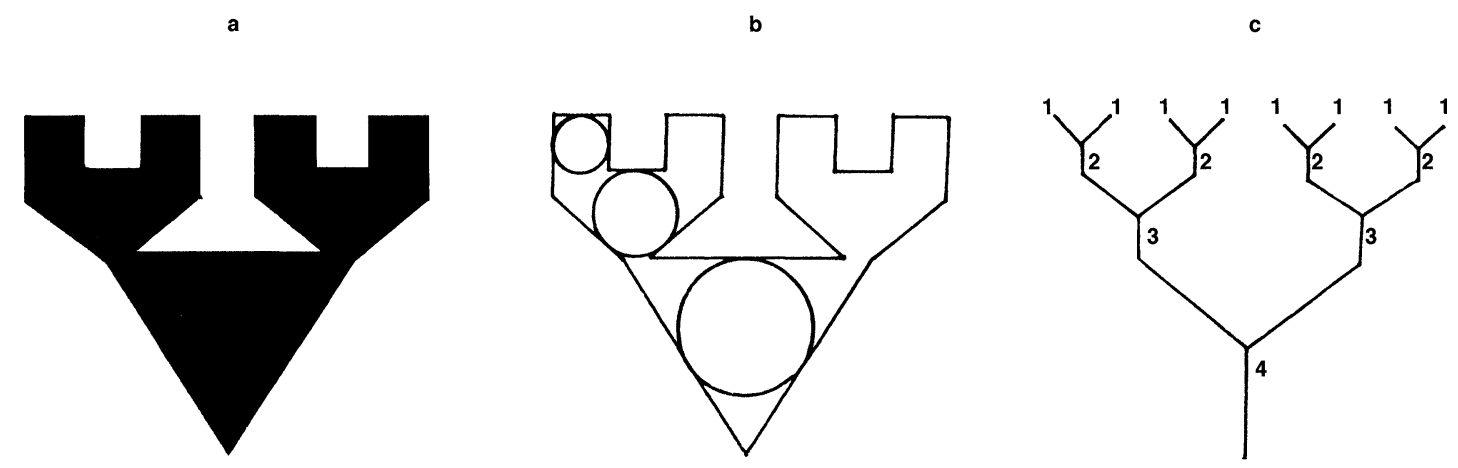

FIGURE 2 (a) Structure, (b) Structure with maximum inscribable circles, and (c) morphological skeleton after designating Strahler's ordering.

(Euclidean space), is defined mathematically as

$$
\begin{aligned}
& \mathrm{CH}_{n}(M)=\left(M \ominus S_{n}\right)-\left\{\left[\left(M \ominus S_{n}\right) \ominus S\right] \oplus S\right\} \\
& \quad n=1,2, \ldots, N, \\
& \mathrm{CH}(M)=\bigcup_{n=0}^{N} \mathrm{CH}_{n}(M),
\end{aligned}
$$

where $\mathrm{CH}_{n}(M)$ denotes the $n$th channel subset of fractal basin $(M)$. In the above expression, subtracting from the eroded versions of $M$ their opening by $S$ retains only the angular points, which are points of the channel in this model. The union of all such possible channel points produces F-SCNs. Depending on the skeletonization process, which determines channel network, numerous types of channel networks ranging from homogeneous to heterogeneous by changing the type of structuring element $(S)$ from regular to irregular are observed.

Though the basins are homogeneous, due to geologic controls the heterogeneous channel network may arise. The rule adopted here is deterministic, non-random type to generate fractal basin. This simplistic model generate river network patterns more closely to realistic networks by considering the asymmetric initiator basins and the random rules to generate fractal basins. Most of the dendritic patterns generally arise in triangular initiator basins. However, in the case study provided, the outlet is at the glory hole (center). Hence, the resultant channel networks are of radial type. As the structural composition of channel network depends on the type of boundary constraints, the boundary choice of the initiator basin becomes vital besides the generating mechanism and the rule for simulating the channel network. The generation of more realistic river networks is possible by considering the natural watershed, which is more often asymmetric, in approximated polygonal form.

\section{FRACTAL BASINS WITH CENTER AS OUTLET, "GLORY HOLE"}

The case study is shown by considering four regular sided initiator-basins, ranging from pentagon to octagon, and a generator (Fig. 3). Considering this generating rule, four initiator-basins were transformed as fractal basins in closed form with the center as outlet ('glory hole'). The channel networks of the fractal basins thus extracted by the procedure detailed in F-SCN model are shown in Figs. 4(a)-(d). These are referred to as five sided to eight sided channel networks as they are extracted from the fractal basins that are generated by considering initiator-basins in the form of regular sided polygons. Since, the considered generator (Fig. 3) is such that it transforms the initiatorbasin, with non-random rule, the following equations hold good as long as the generator, rule, and the regular sided polygons $(Q>4<\infty, Q$ being the 


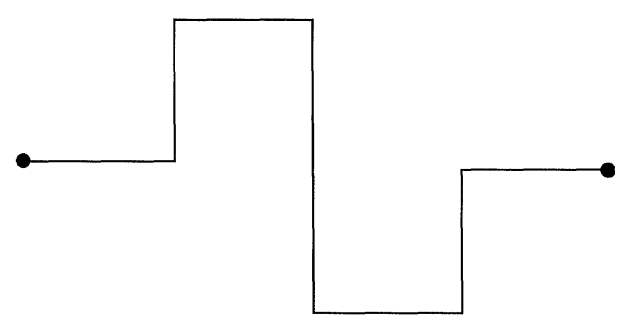

FIGURE 3 Generator.
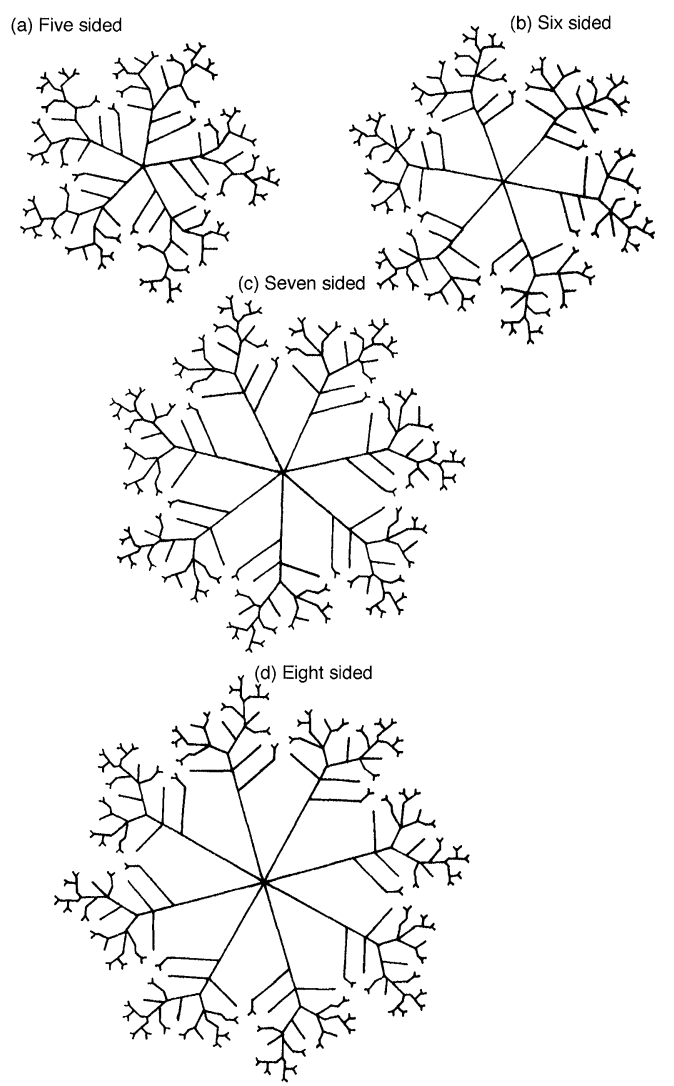

FIGURE 4 Morphological skeletons of the fractal structures.

number of sides of the polygon) are same as the ones considered here.

\section{Morphometric Order Ratios}

Following Strahler's (1957) ordering technique, the extracted channel networks were designated with orders. The basic measures such as area, perimeter of the basins, order-wise channel lengths and number, main channel lengths and total channel lengths, are computed (Table I). It is opined by Beer and Borgas (1993) that the channel density varies with the changing scale in Horton systems. To test the Hortonity of the channel network generated through F-SCN model, the channel density of the fractal basin at different resolutions is computed and it is inferred that the channel network produced through F-SCN is of Horton type.

The morphometric order ratios were computed. The bifurcation ratios were computed as 2.98 for the channel networks shown in Figs. 4(a)-(d), and the length ratios are respectively 2.66, 2.85, 3.09, and 3.41 (Table II). The circularity ratio $\left(R_{C}\right)$ for the considered initiators were computed as 0.8691 , $0.9114,0.937$, and 0.953 respectively. It was observed that, with increasing circularity ratio, the ratio of logarithms of bifurcation ratio, and length ratio decreases, while with increasing circularity ratios, the length ratio increases. No variation was observed in the bifurcation ratio, with the variation in the fractal basin. This is due to the fact that the generating mechanism and rule are the same to transform all the considered initiatorbasins.

The scaling properties of the network as a whole is viewed as the product of the structural composition of the network system and the effect of small irregularities reflected by $d$. Just as in the case of computing the fractal dimension of a river network which depends on its $R_{\mathrm{B}}$ and $R_{\mathrm{L}}$, the fractal dimension of F-SCNs was computed using the three morphometric order ratios, and length-area measures (Table III). Considering these basic morphometric order ratios, several conclusions in relation to other fractal dimensions of fractal basin channel network, and main channel length, computed through box counting method (Feder, 1988), were drawn. The following relationships among morphometric order ratios (Table IV), fractal dimension of basin (Table V), length-area measures (Table III), and certain values arrived at by statistical relations. 
TABLE I Basic measures of morphological skeletons of second order fractal structures

\begin{tabular}{|c|c|c|c|c|c|c|c|c|c|c|c|c|c|c|c|c|}
\hline \multirow[t]{2}{*}{ Initiator } & \multirow[t]{2}{*}{$\begin{array}{c}\text { Area, A } \\
\left(\mathrm{cm}^{2}\right)\end{array}$} & \multirow[t]{2}{*}{$\begin{array}{l}\text { Perimeter, } \\
P(\mathrm{~cm})\end{array}$} & \multirow[t]{2}{*}{$\begin{array}{l}\text { Main } \\
\text { length } l \\
(\mathrm{~cm})\end{array}$} & \multicolumn{4}{|c|}{ No. of orders $N$} & \multicolumn{4}{|c|}{$\begin{array}{l}\text { Mean length of } \\
\text { individual order } \\
(L) \text { (in } \mathrm{cms} \text { ) }\end{array}$} & \multicolumn{4}{|c|}{$\begin{array}{l}\text { Mean areas of individual } \\
\quad \text { order }(A)\left(\text { in } \mathrm{cm}^{2}\right)\end{array}$} & \multirow[t]{2}{*}{$\begin{array}{c}\text { Total } \\
\text { channel } \\
\text { length }(\mathrm{cm})\end{array}$} \\
\hline & & & & 1 & 2 & 3 & 4 & 1 & 2 & 3 & 4 & 1 & 2 & 3 & 4 & \\
\hline Five sided & 110 & 160 & 16 & 130 & 50 & 15 & 5 & 0.56 & 1.1 & 1.67 & 7.5 & 0.313 & 1.21 & 2.78 & 14.06 & 190.25 \\
\hline Six sided & 166.32 & 192 & 18 & 156 & 60 & 18 & 6 & 0.46 & 1.05 & 1.5 & 7.25 & 0.213 & 1.102 & 2.26 & 13.33 & 205.5 \\
\hline Seven sided & 232.63 & 224 & 22 & 182 & 70 & 21 & 7 & 0.65 & 1.3 & 1.67 & 10 & 0.42 & 1.7 & 2.78 & 28.57 & 313.6 \\
\hline Eight sided & 309.1 & 256 & 26 & 208 & 80 & 24 & 8 & 0.54 & 1.26 & 1.67 & 11 & 0.29 & 1.6 & 2.78 & 30.37 & 341 \\
\hline
\end{tabular}

TABLE II Certain order ratios of morphological skeletons of second order fractal structures

\begin{tabular}{|c|c|c|c|c|c|c|c|c|c|c|c|c|c|c|c|}
\hline \multirow{3}{*}{$\begin{array}{l}\text { Initiator- } \\
\text { basin }\end{array}$} & \multicolumn{4}{|c|}{ Bifurcation ratio } & \multicolumn{4}{|c|}{ Length ratio } & \multicolumn{4}{|c|}{ Area ratio } & \multirow{3}{*}{$\begin{array}{l}\text { Circularity } \\
\text { ratio }\left(R_{\mathrm{c}}\right)\end{array}$} & \multirow[t]{3}{*}{$\rho$} & \multirow[t]{3}{*}{$F$} \\
\hline & 2 nd & $3 r d$ & 4 th & \multirow{2}{*}{$\begin{array}{l}\text { Average, } \\
-\quad\left(R_{\mathrm{B}}\right) \\
\end{array}$} & 2nd & $3 \mathrm{rd}$ & 4th & \multirow{2}{*}{$\begin{array}{c}\text { Average, } \\
R_{\mathrm{L}}\end{array}$} & 2 nd & $3 r d$ & 4th & \multirow{2}{*}{$\begin{array}{l}\text { Average, } \\
\left(R_{\mathrm{A}}\right)\end{array}$} & & & \\
\hline & $N_{1} / N_{2}$ & $N_{2} / N_{3}$ & $N_{3} / N_{4}$ & & $L_{2} / L_{1}$ & $L_{3} / L_{2}$ & $L_{4} / L_{3}$ & & $A_{2} / A_{1}$ & $A_{2} / A_{3}$ & $A_{3} / A_{4}$ & & & & \\
\hline Five sided & 2.6 & 3.33 & 3 & 2.98 & 1.96 & 1.52 & 4.5 & 2.66 & 3.87 & 2.3 & 5.06 & 3.74 & 0.8691 & 1.73 & 1.82 \\
\hline Six sided & 2.6 & 3.33 & 3 & 2.98 & 2.28 & 1.43 & 4.83 & 2.85 & 5.17 & 2.05 & 5.9 & 4.37 & 0.9114 & 1.24 & 1.45 \\
\hline Seven sided & 2.6 & 3.33 & 3 & 2.98 & 2 & 1.29 & 5.99 & 3.09 & 4.05 & 1.63 & 10.3 & 5.32 & 0.93656 & 1.35 & 1.21 \\
\hline Eight sided & 2.6 & 3.33 & 3 & 2.98 & 2.33 & 1.33 & 6.6 & 3.41 & 5.51 & 1.74 & 10.9 & 6.06 & 0.953 & 1.1 & 1.04 \\
\hline
\end{tabular}

TABLE III Length-area measures

\begin{tabular}{lll}
\hline Initiator & $l \sim A^{\alpha}$ & $L \sim A^{\beta}$ \\
Five sided & $\alpha=0.59$ & $\beta=1.112$ \\
Six sided & $\alpha=0.563$ & $\beta=0.04$ \\
Seven sided & $\alpha=0.566$ & $\beta=1.055$ \\
Eight sided & $\alpha=0.566$ & $\beta=1.0132$ \\
\hline
\end{tabular}

TABLE IV Fractal dimensions of F-SCN's according to the derivations proposed by hydrogeomorphologists

\begin{tabular}{lcclccc}
\hline Initiator & $D_{1}$ & $D_{2}$ & $D_{3}$ & $D_{4}$ & $D_{5}$ & $D_{6}$ \\
\hline Five sided & 1.1161269 & 1.305 & 1.48 & 2 & 1.74 & 1.25 \\
Six sided & 1.0426 & 1.183 & 1.422 & 2 & 1.78 & 1.18 \\
Seven sided & 1 & 1.09 & 1.36 & 2 & 1.77 & 1.15 \\
Eight sided & 1 & 1.03 & 1.36 & 2 & 1.92 & 1.06 \\
\hline
\end{tabular}

TABLE V Fractal dimensions of fractal structure, morphological skeleton, and main skeletal length

\begin{tabular}{lccc}
\hline Initiator & \multicolumn{3}{c}{ Measured fractal dimensions through box counting method } \\
\cline { 2 - 4 } & $D$-Fractal basin & $D_{\text {Ts-Channel network }}$ & $d$-Main channel length \\
\hline Five sided & 1.72 & 1.63 & 1.16 \\
Six sided & 1.77 & 1.66 & 1.13 \\
Seven sided & 1.81 & 1.7 & 1.13 \\
Eight sided & 1.85 & 1.77 & 1.14 \\
\hline
\end{tabular}


Fractal Relation with Morphometric

Order Ratios $\left(R_{\mathrm{B}}, R_{\mathrm{L}}\right.$, and $\left.R_{\mathrm{A}}\right)$, and Length-Area Measures

The fractal dimensions of fractal basins $(D)$, their channel networks $\left(D_{\mathrm{TS}}\right)$, and main channel lengths (d) are computed through box counting method (Table V). This method provided the values of $D$, $D_{\mathrm{TS}}$, and $d_{1}$ as the slopes of the straight lines which were fitted to the log-transformed pairs of observed box numbers and box size values. For five-sided channel network, the fractal dimensions of fractal basin, channel network, and main channel length were computed by box counting method as 1.72 , 1.63 , and 1.18 respectively. The results for the six sided network respectively are $1.77,1.66$, and 1.13. For seven sided channel network the fractal dimensions are $1.81,1.7$, and 1.132. Table V also shows
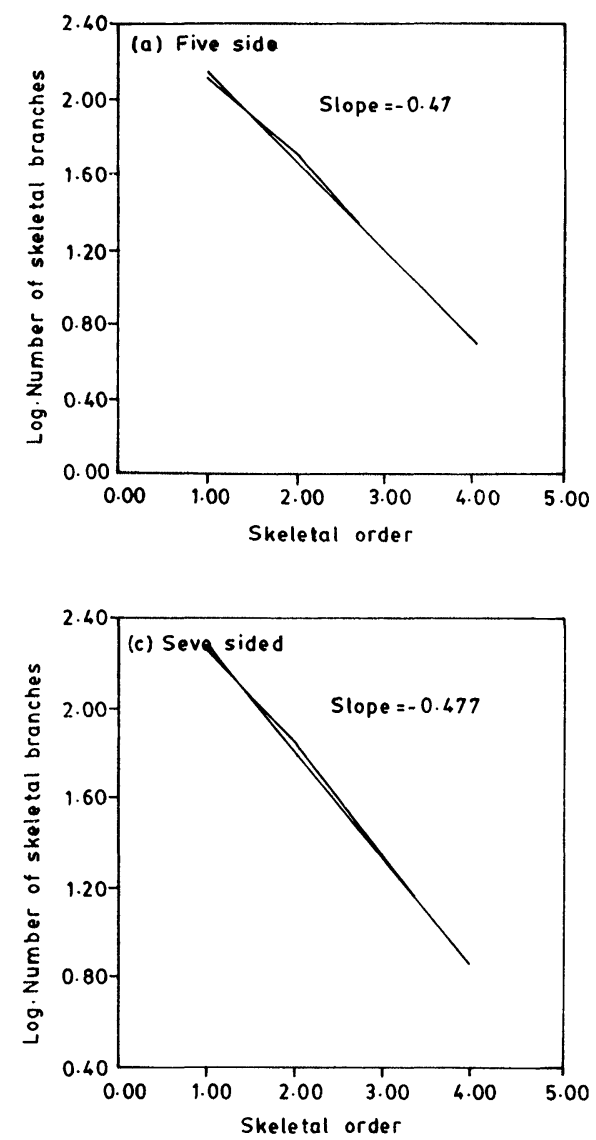

these fractal dimensions, where the initiator-basin is octagon, are $1.85,1.77$, and 1.14 for eight sided channel network. These results were compared with the fractal dimensions arrived at by morphometric order ratios and length-area measures (Tables III and IV). The fractal dimensions computed using morphometric parameters were found valid and they are related to the fractal dimensions computed by box counting method.

\section{Order $(\omega)$ versus Number $N_{\omega=1}^{\Omega} /$ Mean Length} $\bar{L}_{\omega=1}^{\Omega} /$ Mean Area $\left(\bar{A}_{\omega=1}^{\Omega}\right)$

Graphs were plotted between the order number and the logarithms of number of the respective channel branches, and the logarithms of mean channel lengths (Figs. 5(A) and (B)) for the extracted channel networks. The statistical results
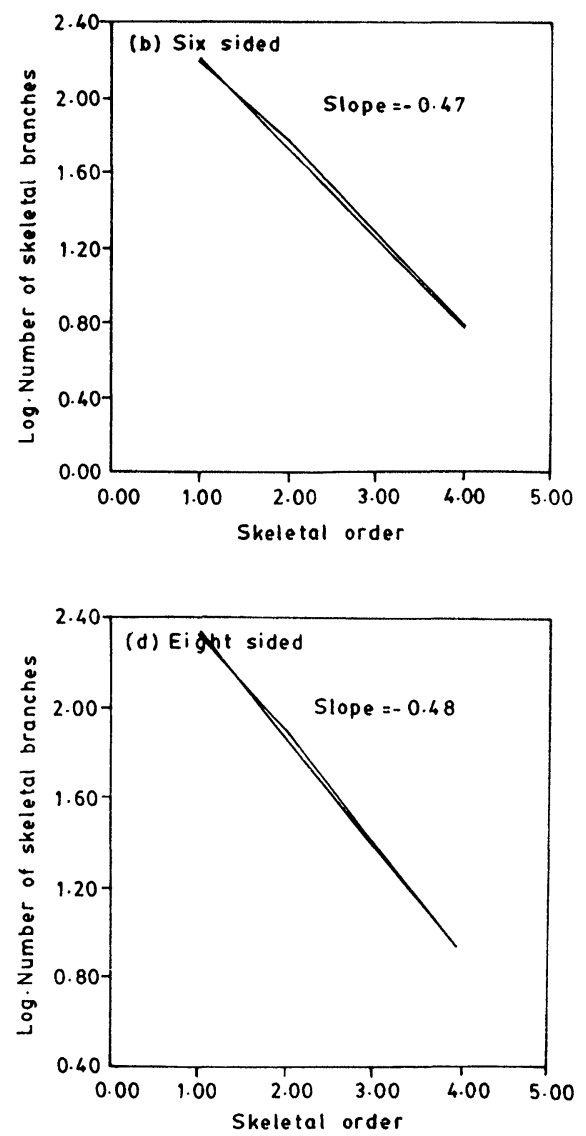

FIGURE 5(A) 

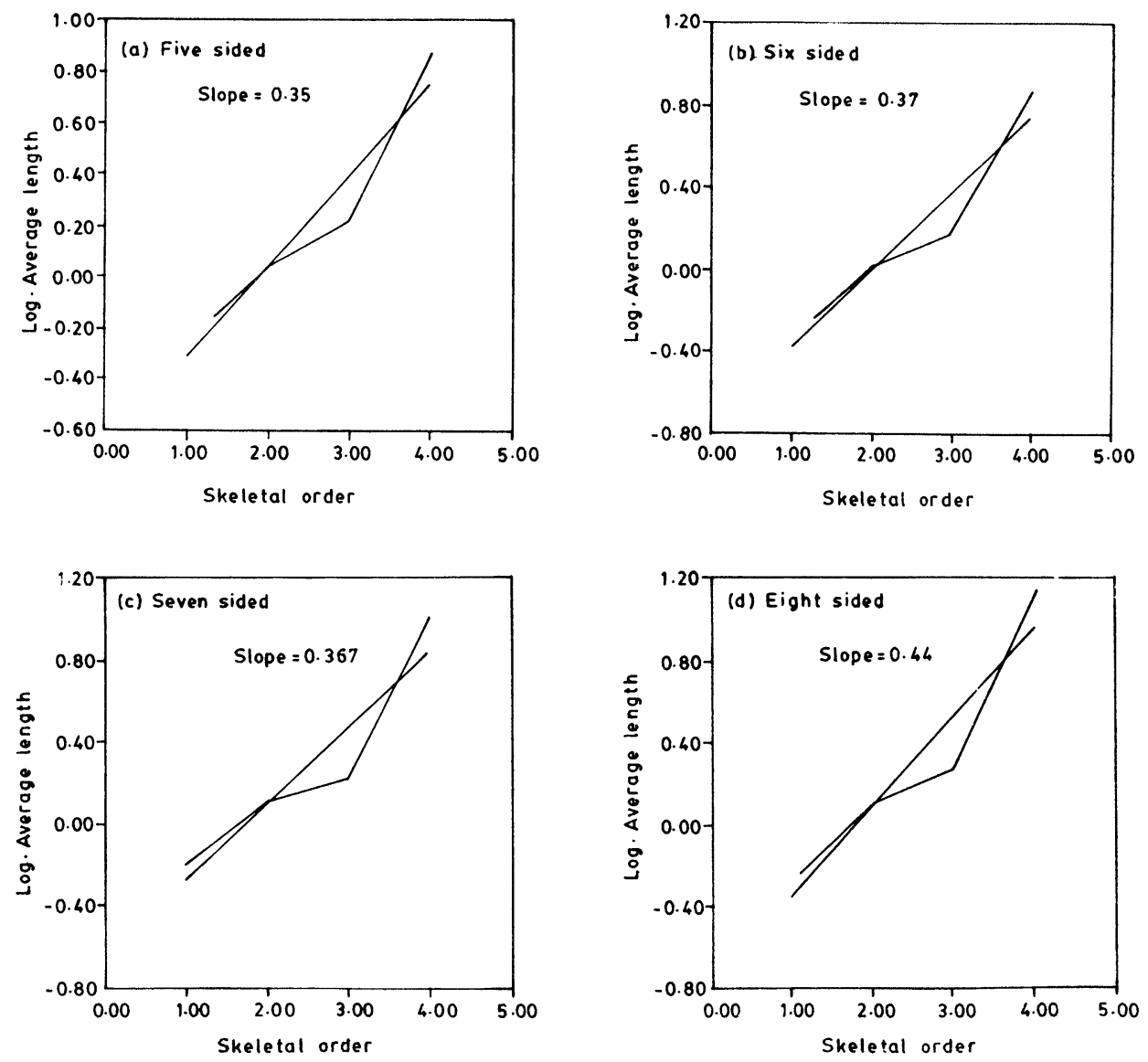

FIGURE 5(B)

FIGURE 5 Graphs between (A) order no. versus $\log$ (number of skeleton branches) and (B) order no. versus log(mean length).

of these two types of plots viz. (a) order versus number, (b) order versus mean lengths were arrived at. The slope values for the plots between order and number are -0.48 for all the considered channel network patterns. In contrast, the slope values for the plots between the order and the mean channel lengths are $0.35,0.37,0.37$, and 0.44 for the channel networks of generated fractal basins respectively. For the plot between order versus channel area, the slope values respectively for five, six, seven and eight sided networks are $0.714,0.7512,0.735$, and 0.81 . These values satisfy the Horton (1945) laws. These laws state that natural drainage basins yield a linear relationship. It is observed, in the latter graph, that the slope values increased with increasing circularity ratio of the fractal basins.

Mean Length $\bar{L}_{\omega=1}^{\Omega}$ versus Number $N_{\omega=1}^{\Omega}$

The number-length statistics for these channel networks are shown in Fig. 6, with the number and mean lengths of orders $1-4$, extracted from four types of fractal basins. The slope values are -0.74 , $-0.78,-0.77$, and -0.92 for the four channel networks respectively. The fractal dimensions $\left(D_{5}\right)$ were computed by subtracting these slope values from unit (one). Hence, they are 1.74, 1.78, 1.77, and 1.92. The fractal dimensions of the basins are 

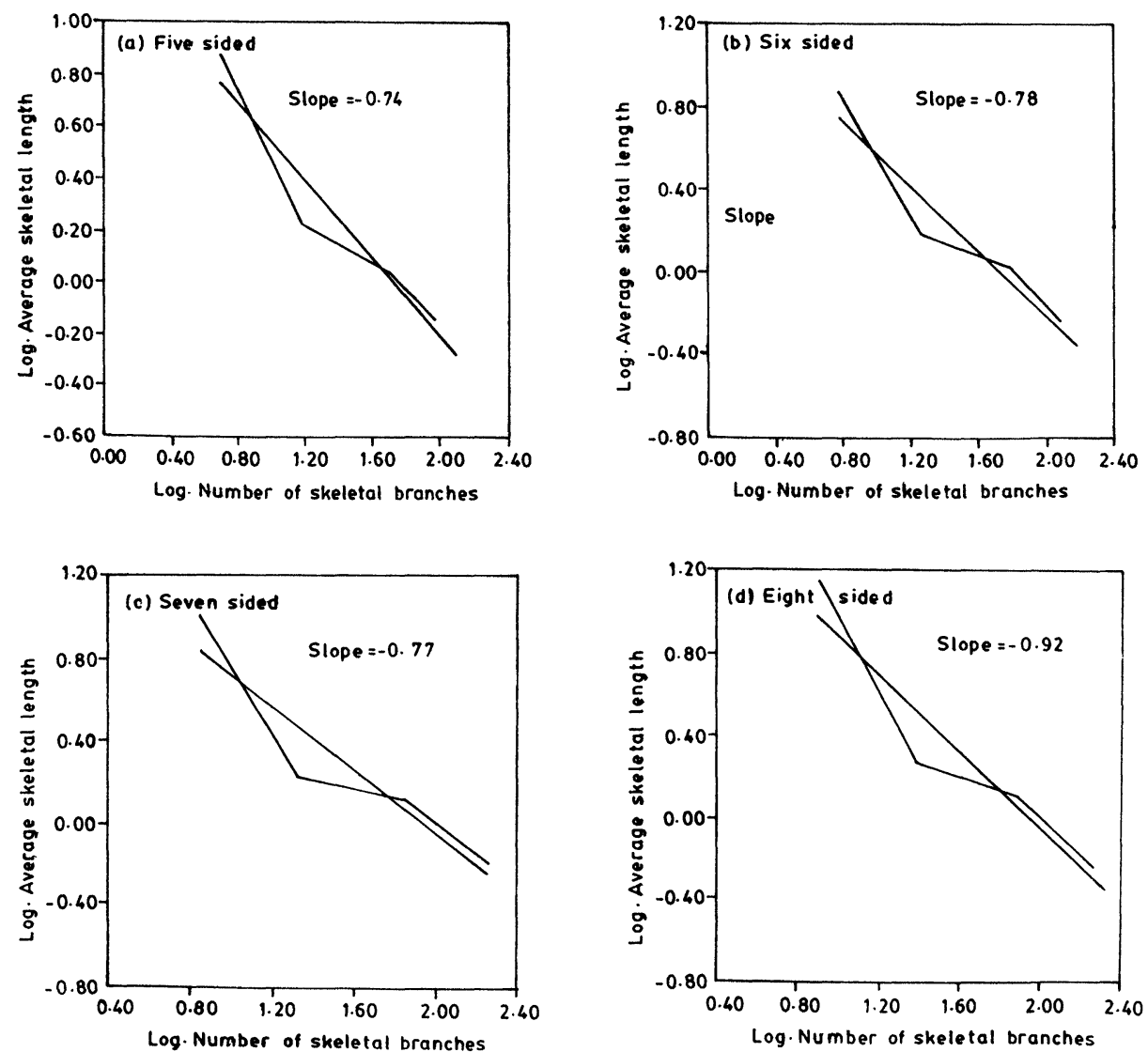

FIGURE 6 Graph showing between $\log$ (no. of skeletons) versus log(average length).

$1.72,1.77,1.81$, and 1.85 respectively (Table V). They are closely tallied with the values arrived at through the statistical plots made between number and mean lengths.

\section{Mean Length $\bar{L}_{\omega=1}^{\Omega}$ versus Total Length $L(\Omega)$}

The plots for logarithms of average branch length versus logarithm of total branch length for these channel networks are shown in Fig. 7. The slope values (b) for these plots respectively for the four channel networks are $-0.25,-0.18,-0.15$, and -0.06 . According to Nelson and Manchester (1988), in the context of lung morphogenesis studies, the fractal dimensions $\left(D_{6}\right)$ were computed as

$$
D_{6}=1-b
$$

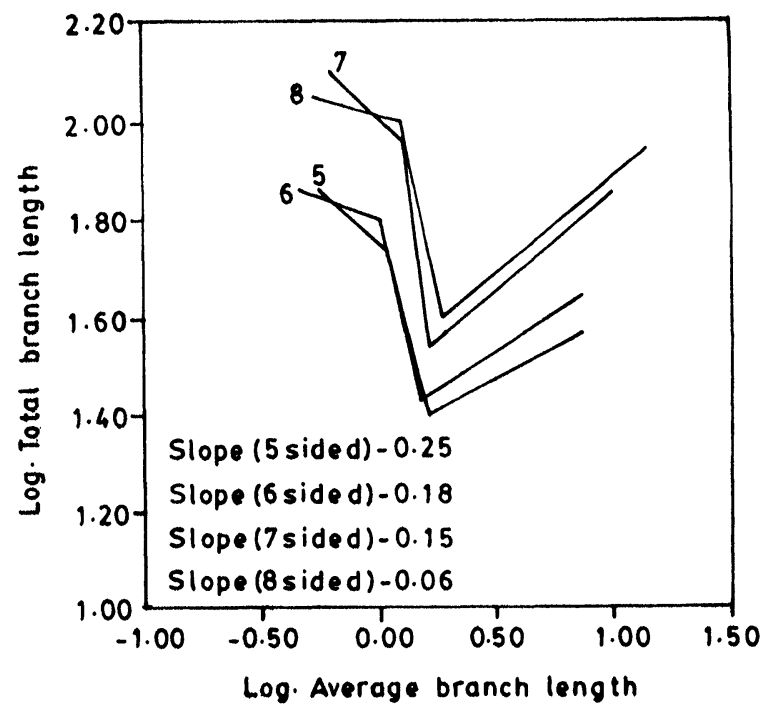

FIGURE 7 Graph showing between log(average branch length) versus $\log$ (total branch length). 
where $D_{6}$ is computed as $1.25,1.18,1.15$, and 1.06 for these four types of F-SCNs. These fractal dimensions are related to the fractal dimensions $\left(D_{2}\right)$, proposed by Tarbotan et al. (1990), in the context of river networks:

$$
D_{6}=D_{2}=d\left(\log R_{\mathrm{B}} / \log R_{\mathrm{L}}\right)
$$

The values arrived at through the equation proposed by Tarbotan et al. (1990), are 1.3, 1.18, 1.1. and 1.03 respectively (Table IV). These values closely tally with the fractal dimensions computed (Fig. 7) between the mean and the total channel lengths. It is apparent that while the overall channel length decreased upto third order, little variations were observed in these plots (Fig. 7) between the third and the forth orders. It was observed that the variation between the $3 \mathrm{rd}$ and 4 th orders is due to the overall structural composition. The fractal dimensions of the four types of channel networks range from 1.06 to 1.25 . These values served as useful parameters to evaluate basin generating algorithm based on the total and average channel lengths. The difference may be observed in the relationship between the average length and the total branch length with the variation either in the initiator-basin, generating mechanism, or in the rule.

\section{Main Channel Length $l(\Omega)$-Area $A(\Omega)$ Relationship}

The important aspect of fractality is concerned with the measured lengths of irregular lines, which yields the length of the channel network to vary with iteration. Dimensional analysis indicates that for geometrically similar network, the length of the main channel, $l$, is proportional to the square root of the area, $A$, is

$$
l \sim A^{1 / 2}
$$

This is also derived as an asymptotic result from the random model of channel networks (Mesa and Gupta, 1987). The empirical relationship between the observed main length, $l$, and the area of fractal basin, $A$, is taken in the form (9) where $\alpha$ is a fitted exponent. The present investigations yield estimated $\alpha$ values, larger than $1 / 2$ for four types of channel networks. This $\alpha$ values range from 0.56 to 0.59 (Table III). As in the case of river length (Mandelbrot, 1982), the main channel lengths of these F-SCNs were also viewed as a fractal with dimension $2 \alpha$. By using four possibilities, it has been found that the fractal dimension for length measurement vary from 1.13 to 1.18 (Table III). As long as the initiator-basin, the generating mechanism and the rule are the same, these results hold good. It is inferred that the fractal dimension of channel length may be related to the structure of network composition. The fractal length-area relationship has been shown as $2 \alpha=d$, where $\alpha$ is an exponent fitted, and $d$ is the fractal dimension of the main channel length computed through box counting method. The difference in exponents may be due to lack of similarity.

\section{Total Length $L(\Omega)$ and Area $A(\Omega)$}

The relationship between the total channel length, $L$, and the area of fractal basin, $A$, was taken in the form of (10) where $\beta$ is a fitted exponent. The present investigation yield estimated $\beta$ values ranging from 1.013 to 1.11 (Table III) for the channel networks extracted from respective fractal basins. These values $(\beta)$ are closely tallied with that of the fractal dimensions computed through computing the ratio of logarithm of $R_{\mathrm{B}}$ and the logarithm of $R_{\mathrm{L}}$. It is related from Eqs. (10) and (12) as follows:

$$
\beta=\left[\log R_{\mathrm{B}} / \log R_{\mathrm{L}}\right] \pm 0.04 .
$$

The relationships between the standard fractal dimensions and the ratios of certain order ratios are liable to vary with the variation either in the generating mechanism or in the initiator-basin. If the similar initiator-basin of any size is transformed by a specific generator, the relationship between the fractal dimensions and the morphometric order ratios is invariant. 
In this case study, certain graphical plots were fitted with a straight line. This was to verify the relationships between the order versus number and certain linear aspects of F-SCNs and through the graphical plots that were proposed in the studies of realistic river morphometric relationships. The slope values computed through these graphical plots tallied with the values proposed in the context of natural river networks inspite of the fact that the plots in the present model did not fit well by straight lines. Moreover, the apparent oscillation about the fit line is a consequence of abrupt variation in the residues corresponding to the middle order branch in the radial type of F-SCNs.

\section{Fractal Relations among Fractal Dimensions of Basins and their Channel Networks}

The fractal measures $D_{1}, D_{2}, D_{3}$, and $D_{6}$, computed through the derivations given in Eqs. (11)-(13) and (19), were directly proportional to each other:

$$
D_{1}=p D_{2}=q D_{3}=r D_{6},
$$

where $p, q$, and $r$ are constants.

The following is an Eq. (22) to describe the relationships between the fractal dimensions of the fractal basin $(D)$, its channel network $\left(D_{\mathrm{TS}}\right)$, the main channel length $(d)$, the ratios of morphometric order ratios of channel networks, the product of generator specifications, and the circularity ratio of the initiator-basin arrived at through statistical analysis

$$
\begin{gathered}
D \sim\left[\left(D_{\mathrm{TS}}\right)^{0.87}+0.32\right] \sim d\left[\frac{\log \left(R_{\mathrm{B}}\right)}{\log \left(R_{\mathrm{L}}\right)}\right]^{-0.454} \\
+2.3 \sim\left[\frac{\log (N)}{\log (1 / r)} R_{c}\right]^{0.986}+0.43 .
\end{gathered}
$$

Highly symmetric initiator-basin with infinite number of equal sides is very close to a circle, of which the circularity ratio is very close to 1 . If such an initiator-basin is transformed by a generating mechanism, according to a non-random rule, as a fractal basin of which the area remains constant throughout the succession of changes, the following relation is proposed.

This relation is established by considering the specification of generating mechanism and circularity ratio of initiator-basins $(4<Q<\infty)$, and fractal dimensions of basins, their channel networks and certain morphometric order ratios of channel networks:

$$
\begin{aligned}
D & \sim\left[\frac{\log (N)}{\log (1 / r)} R_{c}\right]^{0.986}+0.43 \\
& \sim\left[\frac{\log (N)}{\log (1 / r)} R_{c}\right]^{1.024}+0.264 .
\end{aligned}
$$

According to the above expressions for the F-SCNs generated from a regular sided initiator-basin, with infinite number of sides, very close to a circle, the fractal dimensions of the fractal basin, and its corresponding channel network converge to the values 1.92 and 1.77 respectively. Interestingly, the latter value i.e., 1.77 , is the fractal dimension proposed for diffusion limited aggregate.

\section{CONCLUSIONS}

Fractal-skeletal based channel network model (F-SCN) was generated by considering two deterministic quantities in this paper. They are initiatorbasins and a generating mechanism. Moreover, the initiator-basins considered in this study were regular, bounded, and symmetric. The generating mechanism was considered such that it preserved the area of the watershed (fractal basin) under the succession of changes. The rule followed to transform the initiator-basin into fractal basin was of non-random type. As a result of this, and because the characteristics of considered initiator basins are of ideal type, the study is a deterministic one. This model emphasised to describe how the boundary of the basin forces the channel network development. This model also generates channel pattern similar to the realistic networks. The statistical features of this model agreed well with 
the actual networks. The F-SCNs yielded an almost perfect match with well-known empirical geomorphological results and explain the most important structural characteristics observed in the geomorphology of channel networks.

It was inferred that with the variations either in the initiator-basin or in the generating mechanism, the relationship between the fractal dimensions and the order ratios hold differently from each other. In this investigation, the variation was shown as increment in the number of sides of the regular sided initiator-basin ranging from pentagon to octagon. This slight variation, which can be quantified as circularity ratio, in the initiator has not shown much impact in the relationships that have been shown. But, the fractal dimension of the channel network is directly proportional to its basin fractal dimension. As opined by Rigon et al. (1993) in the context of studying the frame work of river basin morphology based on OCNs, in the present F-SCN model, the boundary of the basin clearly forced on the development of channel networks. It is interesting to study the role of the shape of the basin in the optimal structure of channel network. It is suggested to use the initiatorbasin such as triangle and square with one of the vertices as outlets to generate more realistic river networks.

It was an attempt to study the potential application of the blend of fractal geometry and mathematical morphology to develop algorithms for modelling the drainage morphogenisis. The utility of this approach explores the developmental and the morphometric hypothesis with varying boundary conditions of initiator basins, and the specifications of the generating mechanism. The results of these simulations may improve our understanding of known developmental alterations.

\section{Acknowledgements}

The authors are grateful to the reviewers for their comments and constructive criticism. The authors are also grateful to Prof. V.R.R.M. Babu for his valuable suggestions. The first author is grateful to the Department of Science and Technology, India, for the financial support under the grant no. SR/ SY/A-06/1994. The second author is grateful to University Grants Commission, India, for a research associate fellowship.

\section{References}

[1] Beer, T. and Borgas, M. (1993). Horton's laws and the fractal nature of streams. Water Resources Research 29(5), $1457-1487$.

[2] Blum, H. (1973). Biological shape and visual sciences (Part I). Journal Theoretical Biology 38, 205-287.

[3] Feder, J. (1988). Fractals (Plenum, New York).

[4] Hack, J.T. (1957). Studies of longitudinal profiles in Virginia and Maryland. U.S. Geol. Surv. Prof. Pap., 294B.

[5] Hadwiger, H. (1957). Vorksungen uber Inhalt, Oberflache and Isoprimetrie (Berlin, Germany: Springer Verlag).

[6] Horton, R.E. (1945). Erosional development of stream and their drainage basin: hydrological approach to quantitative morphology. Bulletin of the Geophysical Society of America 56, 275-370.

[7] La Barbera, P. and Rosso, R. (1987). Fractal geometry of river networks. Eos. Transactions. AGU 68(44), 1276.

[8] La Barbera, P. and Rosso, R. (1989). On the fractal dimension of stream networks. Water Resources Research 25(4), 735-741.

[9] Lantuejoul, C. (1978). La sequelettisation et son application aux mesures topologuiques des mosaiques polycristallines, These de Docteur-lngnieur, School of Mines, Paris, France.

[10] Lantuejoul, C. (1980). Skeletonization in quantitative metallography. In Issues of Digital Image Processing, eds. R.M. Haralick and J.C. Simon (Groningen, The Netherlands: Sijthoff and Neordhoff).

[11] Mandelbrot, B.B. (1982). Fractal Geometry of Nature (Freeman \& Co., San Francisco).

[12] Maragos, P.A. and Schafer, R.W. (1986). Morphological skeleton representation and coding of binary images. IEEE Transactions on Acoustics Speech and Signal Processing ASSP-34, 5.

[13] Marani, A., Rigon, R. and Rinaldo, A. (1991). A note on fractal channel networks. Water Resources Research 27(12), 3041-3049.

[14] Masek, J. and Turcotte, D.L. (1993). A diffusion aggregation model for the evolution of drainage networks. Earth and Planetory Science Letters 119, 379-386.

[15] Mesa, O.J. and Gupta, V.K. (1987). On the main channel length-area relationship for channel networks. Water Resources Research 23(11), 2119-2122.

[16] Nelson, T.R. and Manchester, D.K. (1988). Modeling of morphogenesis using fractal geometries. IEEE Transactions on Medical Imaging 7(4), 321-327.

[17] Rigon, R., Rinaldo, A., Rodrguez-lturbe, I., Bras, R.L. and Ijjasz-Vasquez, E. (1993). Optimal channel networks a framework for the study of river basin morphology. Water Resources Research 29(6), 1635-1646.

[18] Rosso, R., Becchi, B. and LaBarbera, P. (1991). Fractal relation of main stream length to catchment area in river networks. Water Resources Research 27(3), 381-387. 
[19] Sagar, B.S.D. (1996). Fractal relation of a morphological skeleton. Chaos, Solitons, Fractals 7(11), 18711879.

[20] Schumm, S.A. (1956). Evolution of drainage systems and slopes in badlands at Perth Amboy, NJ. Bulletin of Geological Society of America 67, 597-646.

[21] Serra, J. (1982). Image Analysis and Mathematical Morphology. Academic Press, New York.

[22] Shreve, R.L. (1967). Infinite topologically random channel networks. Journal Geology 77, 397-414.
[23] Smart, J.S. (1972). Channel networks. Advances in Hydrosciences 8, 305-346.

[24] Stark, C.P. (1991). An invasion percolation model of drainage network evolution. Nature 352, 423-425.

[25] Strahler, A.H. (1957). In Handbook of Applied Hydrology, Sect. 4-11, Ed. V.T. Chow (New York, Mc Graw-Hill).

[26] Tarbotan, D.G., Bras, R.L. and Rodriguez-lturbe, I. (1990). Comment on "On the fractal dimension of stream networks by Paolo La Barbera and Rosso Renzo". Water Resources Research 26(9), 2243-2244. 


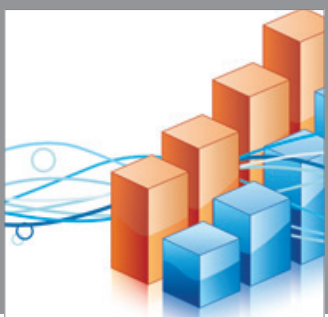

Advances in

Operations Research

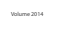

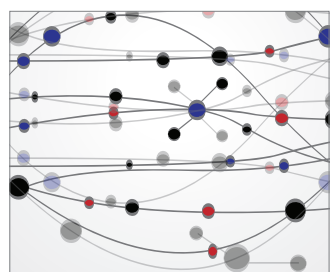

\section{The Scientific} World Journal
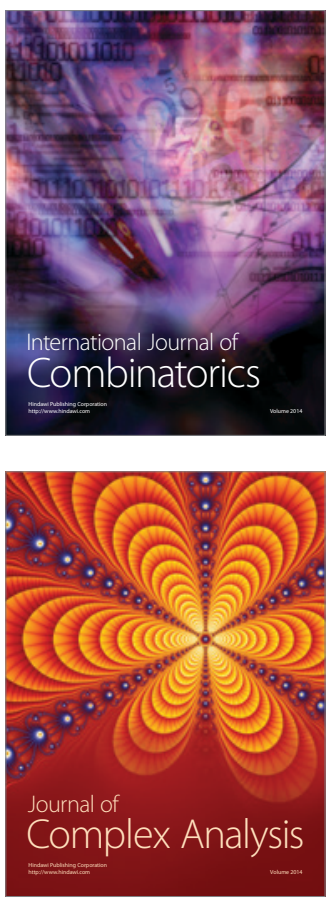

International Journal of

Mathematics and

Mathematical

Sciences
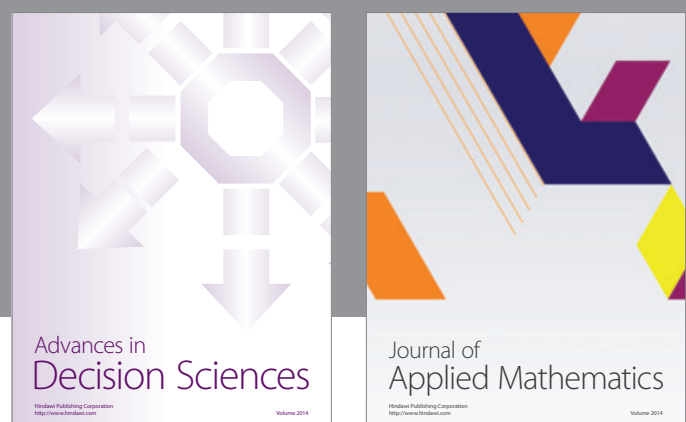

Journal of

Applied Mathematics
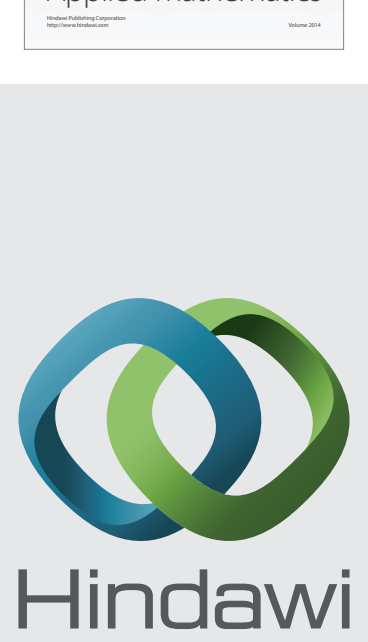

Submit your manuscripts at http://www.hindawi.com
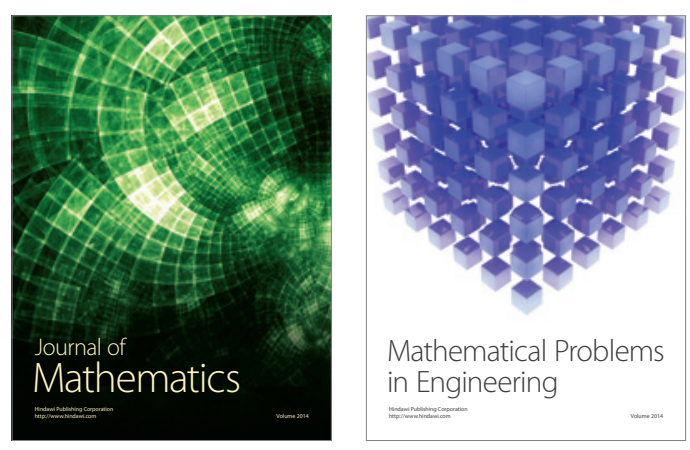

Mathematical Problems in Engineering
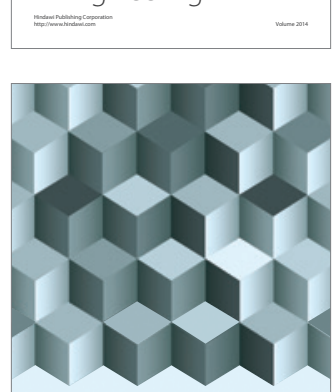

Journal of

Function Spaces
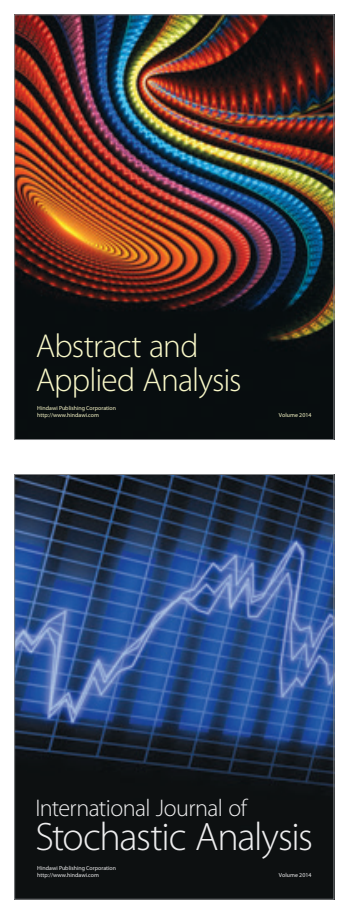

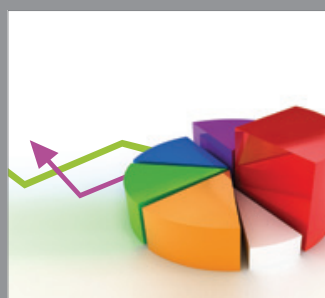

ournal of

Probability and Statistics

Promensencen
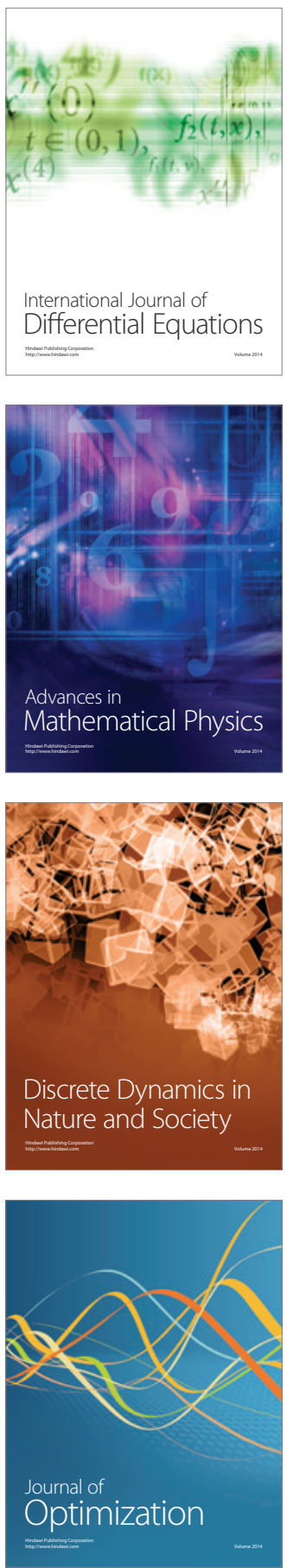